Original Research Paper

\title{
Gender Differences in the Prevalence of Cardiovascular Risk Factors in an Iranian Urban Population
}

\author{
${ }^{1}$ Mohammad Shojaei, ${ }^{1}$ Abdolreza Sotoodeh Jahromi, \\ ${ }^{2}$ Karamatollah Rahmanian and ${ }^{3}$ Abdolhossein Madani \\ ${ }^{I}$ Research Center for Non-Communicable Diseases, Jahom University of Medical Sciences, Jahrom, Iran \\ ${ }^{2}$ Research Center for Social Determinant of Health, Jahom University of Medical Sciences, Jahrom, Iran \\ ${ }^{3}$ Research Center for Social Determinants of Health Promotion, \\ Hormozgan University of Medical Sciences, Bandarabbas, Iran
}

\author{
Article history \\ Received: 22-04-2015 \\ Revised: $14-05-2015$ \\ Accepted: 24-07-2015 \\ Corresponding Author: \\ Karamatollah Rahmanian \\ Research Center for Social \\ Determinant of Health, Jahom \\ University of Medical \\ Sciences, Jahrom, Iran \\ E-mail: rahmaniank47@yahoo.com
}

\section{Introduction}

The Cardiovascular Disease (CVD) is one of the most important causes of mortality in the world and the burden of this disease is increasing in all developing countries (Ebrahsim and Davey-Smith, 2001; Boutayeb and Boutayeb, 2005). Smoking, high blood pressure, lipid disorder, diabetes mellitus (Almdal et al., 2004; Tang et al., 2004; Murphy et al., 2009; Amarenco et al., 2010; Willey et al., 2009), infectious disease such as mycoplasma pneumonia (Pourahmad et al., 2009), low serum adiponectin level (Shojaie et al., 2009a) and inflammation (Jahromi et al., 2010a), low serum level of Annexin-V (Shojaie et al., 2009b) and antiphospholipid antibodies (Jahromi et al., 2013a; 2013b; 2013c; Shojaei et al., 2011; Jahromi et al., 2010b; 2014), are fundamental risk factors of heart disease. In a cohort study, the increased one standard deviation in systolic blood pressure was associated with $35 \%$ increment of risk of CVD (Hadaegh et al., 2011). Also, accumulation of risk factors in one subject increased risk of CVD (Wilson et al., 1987; Aizawa et al., 2004). The high systolic blood pressure was associated with higher risk of CVD mortality (Wang et al., 2009).

Cardiovascular disease is the leading cause of mortality in adults in Iran (Hadi and Rostami-Gooran, 2004). Several studies in Iran showed that the prevalence of CVD risk factors is high in community (Sharifi et al., 2009; Rahmanian and Shojaie, 2011; Bonakdaran et al., 2011). The prevalence of CVD risk factors in Bushehr is high, particularly high truncal obesity, low High-Density Lipoprotein Cholesterol (HDL-C) and high triglyceride (Nabipour et al., 2008). In another study as part of a national survey of noncommunicable diseases from the Khorasan province, northeast of Iran, showed that the prevalence of CVD risk factors, mainly low HDL-C and hypertension were high (Azimi-Nezhad et al., 2009). 
Iran is a developing country that has had rapid changes in life style. Also, the prevalence of hypertension, diabetes mellitus and metabolic syndrome in Jahrom was high, 35.4, 11.6 and $28.8 \%$, respectively (Rahmanian and Shojaie, 2012; 2011; Rahmanian et al., 2013). Then, we determined gender different of CVD risk factors in Jahrom city, Iran.

\section{Materials and Methods}

The adults aged 30 years and over were randomly selected stratified by gender and age from ten urban centers of Jahrom in the south region of Iran and invited to participate in the study. Pregnant and lactating women and/or persons with chronic disease and mental disorders and unable to walk, were excluded. The final sample consisted of 405 males (aged 51.9 \pm 13.9 years) and 487 females (aged $48.5 \pm 12.9$ years).

All subjects (892) answered a demographic and a detailed medical questionnaire. Informed consent was obtained from all adult participants. The research protocol was approved by the Research Committee of Jahrom University of Medical Sciences.

Afterwards, they were subjected to anthropometric, blood pressure and fasting blood sugar and lipid profile measurements. All data were collected during the years 2008 and 2010. All measurements were conducted by a trained physician. Identical standard protocols were used for each measurement explained elsewhere (Rahmanian and Shojaie, 2012).

Body Mass Index (BMI) was categorized as normal (less than 25.0), overweight (25.0-less than 30.0) and obese (30.0 or greater) (WHO, 2000). Hypertension was defined as $140 / 90 \mathrm{mmHg}$ or greater and/or self reported current treatment with antihypertensive drugs (NIH, 2008). High cholesterol was defined as $\geq 240$ $\mathrm{mg} / \mathrm{dL}$ ), high LDL-cholesterol as $\geq 160 \mathrm{mg} / \mathrm{dL}$; low HDL-cholesterol as $<40 \mathrm{mg} / \mathrm{dL}$ for men and $<50 \mathrm{mg} / \mathrm{dL}$ for women and high triglyceride levels as $\geq 200 \mathrm{mg} / \mathrm{dL}$ (EPDETHBCA, 2001; Shojaei et al., 2011).

Participants were diagnosed with diabetes mellitus if they had fasting blood glucose level of $\geq 126 \mathrm{mg} / \mathrm{dL}$, reported a history of diabetes mellitus, or were currently receiving hypoglycemic drugs (ADA, 2006). Subjects, who smoked one or more cigarettes or one cup of water pipe per week, were considered as smokers.

The distribution of Cardiovascular Disease (CVD) risk factors was compared across gender by using the chi square test for the difference between proportions. For continuous CVD risk factors, comparisons were done with the independent t-test.

For categorical CVD risk factors, we used logistic regression to estimate the odds ratio with $95 \%$ confidence interval. In all the analyses, a p-value of $<0.05$ was considered statistically significant. Data analysis was carried out with the SPSS software, version 11.5.

\section{Results}

Descriptive characteristics of participants are summarized in Table 1. Women were significantly younger $(p<0.001)$ and lower married $(p<0.001)$, compared to men. Although, women were lower educated than men but this difference was no significant $(\mathrm{p}=0.053)$.

In comparison to men, women had significantly a higher mean of BMI, T-C, LDL-C and HDL-C but lower mean of TG (Table 2). But, the mean of systolic blood pressure, diastolic blood pressure and fasting blood sugar did not significantly differ by gender.

Women had significantly a higher prevalence of obesity and low HDL-C but had a lower prevalence of smoker in compared to men (Table 3). But the prevalence of hypertension, diabetes mellitus, hypercholesterolemia and elevated of LDL-C and triglyceride levels were not significantly difference. The most common risk factors were low HDL-C and overweight in women and overweight and hypertension in men, respectively.

In logistic regression analyses, the odds of overweight, obesity, smoking, hypertension, elevated triglyceride, hypercholesterolemia and lower HDL-C significantly differed by gender (Table 4).

Table 1. Gender differences in socio-demographic characteristics of participants

\begin{tabular}{llccccl}
\hline Categorized variables & & Male no & $\%$ & Female no & $\%$ & $\mathrm{p}$ \\
\hline Education & Illiterate & 68 & 16.8 & 101 & 20.7 & 0.053 \\
& Diploma or lower & 259 & 63.9 & 324 & 66.6 & \\
Marriage & Higher than diploma & 78 & 19.3 & 62 & 12.7 & \\
& Married & 387 & 95.6 & 411 & 84.4 & $<0.001$ \\
& Single & 14 & 3.5 & 20 & 4.1 & \\
Continues variable & Widow or divorce & 4 & 1.0 & 56 & 11.5 & \\
Age, year & & Mean & SD & Mean & SD & \\
\hline
\end{tabular}


Mohammad Shojaei et al. / OnLine Journal of Biological Sciences 2015, 15 (3): 178.184 DOI: 10.3844/ojbsci.2015.178.184

Table 2. Sex differences in mean of cardiovascular risk factors

\begin{tabular}{|c|c|c|c|c|c|}
\hline Variables & Men mean & SD & Women mean & SD & $\mathrm{p}$ \\
\hline Weight, kg & 71.7 & 12.5 & 66.9 & 12.2 & $<0.001$ \\
\hline Height, $\mathrm{cm}$ & 168.8 & 6.9 & 156.1 & 6.1 & $<0.001$ \\
\hline BMI, $\mathrm{kg} / \mathrm{m}^{2}$ & 25.1 & 3.9 & 27.4 & 4.5 & $<0.001$ \\
\hline SBP, $\mathrm{mmHg}$ & 127.2 & 18.3 & 127.0 & 20.6 & 0.876 \\
\hline DBP, $\mathrm{mmHg}$ & 80.5 & 11.4 & 79.9 & 11.2 & 0.436 \\
\hline $\mathrm{FBS}, \mathrm{mg} / \mathrm{dL}$ & 96.9 & 32.8 & 99.2 & 43.0 & 0.381 \\
\hline $\mathrm{TG}, \mathrm{mg} / \mathrm{dL}$ & 149.9 & 102.1 & 137.1 & 76.3 & 0.032 \\
\hline $\mathrm{T}-\mathrm{C}, \mathrm{mg} / \mathrm{dL}$ & 185.9 & 40.0 & 195.4 & 42.7 & 0.001 \\
\hline LDL-C, mg/dL & 112.8 & 31.0 & 117.8 & 32.4 & 0.019 \\
\hline HDL-C, $\mathrm{mg} / \mathrm{dL}$ & 45.0 & 10.1 & 50.2 & 10.3 & $<0.001$ \\
\hline
\end{tabular}

Table 3. Prevalence of cardiovascular risk factors according to the gender

\begin{tabular}{lccccc}
\hline Variables & Men & $\%$ & Women no & $\%$ & $\mathrm{p}$ \\
\hline Smoker & 108 & 26.7 & 8 & 1.6 & $<0.001$ \\
Overweight & 167 & 41.3 & 216 & 44.4 & $<0.001$ \\
Obesity & 40 & 9.9 & 121 & 24.8 & $>0.05$ \\
Hypertension & 142 & 35.1 & 173 & 35.5 & $>0.05$ \\
Systolic HTN & 109 & 27.0 & 147 & 30.2 & $>0.05$ \\
Diastolic HTN & 109 & 11.1 & 59 & 28.5 & $>0.05$ \\
Diabetes mellitus & 45 & 19.8 & 79 & 12.1 & $>0.05$ \\
High TG & 80 & 9.4 & 65 & 16.2 & $>0.05$ \\
High T-C & 38 & 6.4 & 48 & 13.3 & $>0.05$ \\
High LDL-C & 26 & 32.3 & 250 & 9.9 & $<0.001$ \\
Low HDL-C & 131 & 26.7 & 216 & 1.6 & $<0.001$ \\
Smoker & 108 & 41.3 & 121 & 44.4 & $<0.001$ \\
Overweight & 167 & 9.9 & 173 & 24.8 & \\
Obesity & 40 & 35.1 & 147 & 35.5 & $>0.05$ \\
Hypertension & 142 & 27.0 & 59 & 30.2 & $>0.05$ \\
Systolic HTN & 109 & 27.0 & 79 & 28.5 & $>0.05$ \\
Diastolic HTN & 109 & 11.1 & 65 & 12.1 & $>0.05$ \\
Diabetes mellitus & 45 & 19.8 & 48 & 16.2 & $>0.05$ \\
High TG & 80 & 9.4 & 250 & 13.3 & $>0.05$ \\
High T-C & 38 & 32.4 & & 9.9 & $>0.05$ \\
High LDL-C & 131 & & 51.3 & $<0.001$ \\
Low HDL-C & & & &
\end{tabular}

Table 4. Binary logistic regression analysis of gender and cardiovascular risk factors

\begin{tabular}{llll}
\hline Variables & OR & CI 95\% & $\mathrm{p}$ \\
\hline Normal BMI, reference & 1.00 & $1.04-2.0$ & 0.028 \\
Overweight & 1.44 & $2.03-5.09$ & 0.000 \\
Obesity & 3.22 & $0.02-0.09$ & 0.000 \\
Smoker & 0.04 & $0.28-0.95$ & 0.034 \\
Hypertension & 0.51 & $0.37-0.83$ & 0.005 \\
High triglyceride & 0.55 & $1.40-3.90$ & 0.001 \\
Hypercholesterolemia & 2.34 & $2.11-4.04$ & 0.000 \\
Low HDL-C & 2.92 & & \\
\hline
\end{tabular}

Women had 3.22 times greater odds of being obese and 1.44 times greater odds of overweight. Also, women had 2.92-fold greater risk of having low HDL-C and more than two-fold greater risk of having hypercholesterolemia compared to men. On the contrary, women had more than twenty three-fold lower risk of being smoking, compared to men $(p<0.001)$. Also, women had lower risk of having hypertension and elevated triglyceride level than men (near 50\%).

The prevalence of combinations of CVD risk factors for both genders is displayed in Fig. 1. Totally $11.3 \%$ of women and only $6.9 \%$ of men had no cardiovascular risk factor in the population $(\mathrm{p}=0.002)$. The most of participants had one or two risk factors (50.9\% in women and $51.1 \%$ in men). 


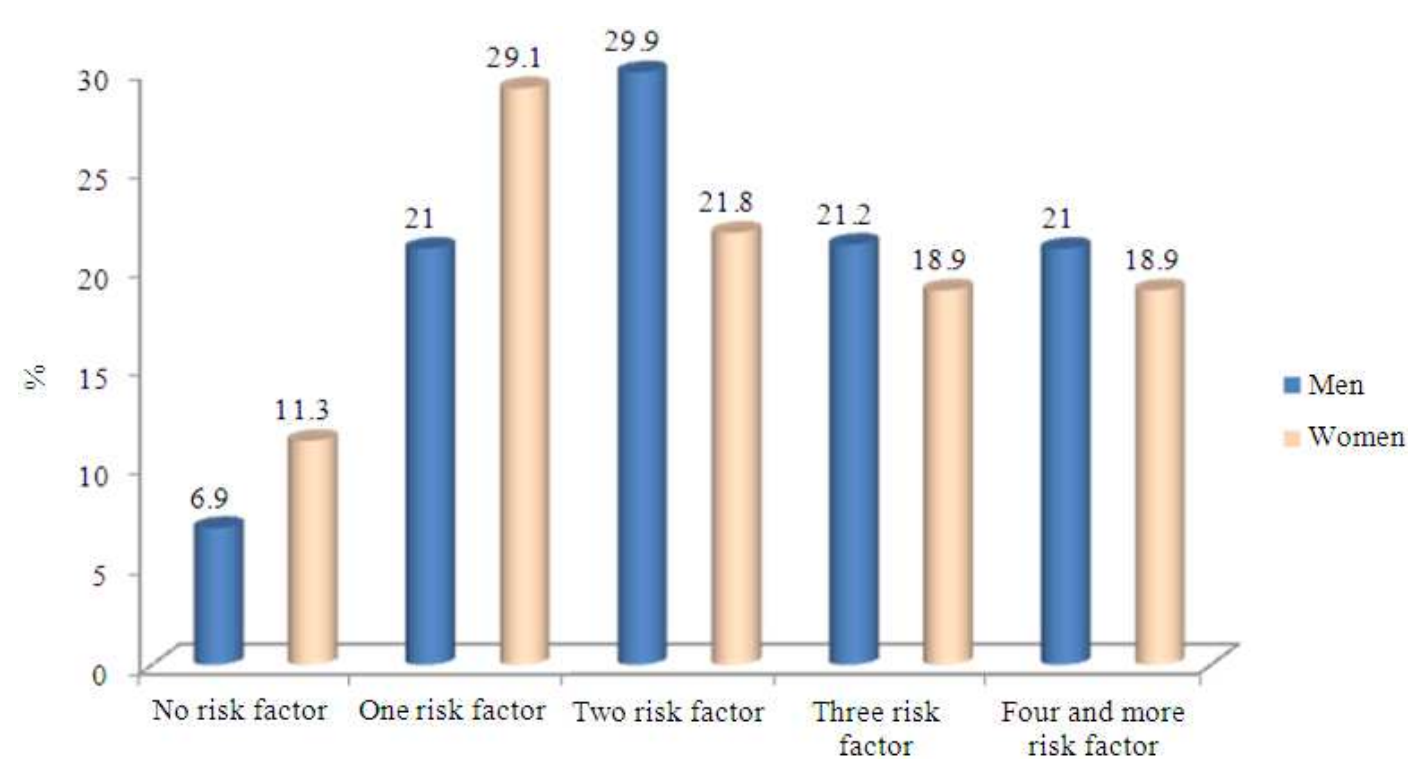

Fig. 1. The frequency distribution of cumulative cardiovascular risk factors among women and men

\section{Discussion}

In this study among adults in an urban south region of Iran, we noted a high prevalence of cardiovascular disease risk factors, including obesity, hypertension, dyslipidemia and diabetes mellitus. Several gender disparity in CVD risk factors were observed, particularly high levels of obesity and low HDL-C among women.

Similar to study conducted by (Bahonar et al., 2011) in Isfahan, Iran, in our study women had significantly higher mean BMI, TC, LDL-C and HDL-C and lower TG level compare to men. In the third National Survey of Risk Factors of Non-Communicable Diseases (SURFNCD 2007), only the mean diastolic blood pressure was lower and fasting blood sugar was higher in urban women than in men (Delavari et al., 2009). In another study in Bushehr, women had significantly higher mean serum TC, LDL-C and HDL-C but lower mean triglyceride and DBP and SBP (Nabipour et al., 2008). In contrast to our study, findings from a study in Tanzania, triglycerides, TC, LDL-C and HDL-C levels did not significantly differ by gender. But Tanzanian women had significantly higher mean BMI and lower systolic and blood pressure level, compared to men (Njelekela et al., 2009). Also, (Wu et al., 2011) in their study conducted in Taiwan reported that women had a lower mean of BMI, SBP, DBP, TC and FBS than in men, but a higher mean of HDL-C. In Addis Ababa, the authors reported a higher mean BMI and lower mean of SBP and DBP (Tesfaye et al., 2009).

In this study, we found that prevalence of obesity was higher in women. This result is in agreement with findings from other studies in Great Tunis (Elasmi et al., 2009), Addis Ababa (Tesfaye et al., 2009), China (Yang et al.,
2012), India (Gupta et al., 2007) and Iran (Nabipour et al., 2008; Bahonar et al., 2011; Delavari et al., 2009). The higher prevalence of obesity among women may be attributed to socio-cultural factors such as work activity and lifestyle. In contrast to our result, others reported higher prevalence of obesity in men (Wu et al., 2011; Latheef and Subramanyam, 2007) than in women.

Our study showed that prevalence of low HDL-C level was higher in women than men. This finding is consistent to other reports from India (Latheef and Subramanyam, 2007) and is different with result of studies from Iran (Nabipour et al., 2008; Bahonar et al., 2011).

Similar to those reported from Mexico (Meaney et al., 2007), Great Tunis (Elasmi et al., 2009), Addis Ababa (Tesfaye et al., 2009), India (Gupta et al., 2007), China (Wu et al., 2011; Yang et al., 2012) and Iran (Nabipour et al., 2008; Bahonar et al., 2011), in the present study, prevalence of smoking significantly was lower in women than in men.

In opposite to our findings, several studies showed that prevalence of high LDL-C (Nabipour et al., 2008; Bahonar et al., 2011), hypercholesterolemia (Gupta et al., 2007; Latheef and Subramanyam, 2007; Meaney et al., 2007) was higher in women than in men but prevalence of hypertension (Wu et al., 2011; Yang et al., 2012), elevated TG was lower in women than in men.

The most important CVD risk factor was low HDL-C in both gender that the percent of this lipid disorders lower than reported in Zanjan, 2002-2003 (Sharifi et al., 2009). Such finding reported by (Nabipour et al., 2008) in PGHHS in Bushehr, Iran. In study conducted by (Bahonar et al., 2011) the major risk factors in older women was hypertension and obesity and in men was hypertension and overweight. In the study conducted by 
Wu et al. (2011) lipid disorder was the major risk factor in men and overweight/obesity was the most prevalent risk factor in women.

In logistic regression analysis, we observed higher odds of overweight, obesity, hypercholesterolemia and low HDL-C level among women compared to men, despite significantly lower rates of smoking, hypertension and elevated triglyceride. In urban Tanzanian women, the odds of overweight, hypercholesterolemia, elevated LDL$\mathrm{C}$ and diabetes mellitus did not significantly differ by gender (Njelekela et al., 2009). But women had 4.3 times greater odds of being obese, compared to men. The comparatively lower rates of hypertension among women could be caused by a protective effect of estrogen and lower rates of smoking compared to men.

The limitation of our study was the cross-sectional sampling design does not permit inferences to be drawn relating to the causal relationships among variables. Also, in this study CV risk factors such as BMI, high serum lipid, blood sugar, blood pressure and other risk factors were not analyzed in participant parents or families.

\section{Conclusion}

In conclusion, this study auxiliary adds strong facts for the high prevalence of cardiovascular diseases risk factors in Iran, particularly in women. Health promotion, primary prevention and health screening strategies are needed to target hypertension, smoking, lipid disorders and obesity.

\section{Acknowledgement}

We extend our gratitude's to all our participants without their cooperation this important data could not have been known.

\section{Funding Information}

This study was supported by research foundation at Jahrom University of Medical Sciences.

\section{Competing Interests}

The authors declare that they have no competing interests.

\section{Authors' Contributions}

All authors had read and approved the final manuscript:

Abdolreza Sotoodeh Jahromi, Karamatollah Rahmanian and Abdolhossein Madani: Designed the study, carried out data collection, analysis and writing the manuscript.
Mohammad Shojaei: Participated in data collection and contributed to the revision of the manuscript.

\section{Ethics}

This article is original and contains unpublished material. The corresponding author confirms that all of the other authors have read and approved the manuscript and no ethical issues involved.

\section{References}

ADA, 2006. American diabetes association: Diagnosis and classification of diabetes mellitus. Diabetes Care, 29: S43-S48.

Aizawa, Y., H. Watanabe, H. Fukunaga and S. Watanabe, 2004. Excess accumulation of risk factors in ischemic heart disease. Jpn. Heart J., 45: 733-738. DOI: 10.1536/jhj.45.733

Almdal, T., H. Scharling, J.S. Jensen and H. Vestergaard, 2004. The independent effect of type 2 diabetes mellitus on ischemic heart disease, stroke and death: A population-based study of 13,000 men and women with 20 years of follow-up. Arch. Intern. Med., 164: 1422-1426.

DOI: $10.1001 /$ archinte.164.13.1422

Amarenco, P., L.B. Goldstein, H. Sillesen, O. Benavente and R.M. Zweifler et al., 2010. Coronary heart disease risk in patients with stroke or transient ischemic attack and no known coronary heart disease: Findings from the Stroke Prevention by Aggressive Reduction in Cholesterol Levels (SPARCL) Trial. Stroke, 41: 426-430. DOI: 10.1161/STROKEAHA.109.564781

Azimi-Nezhad, M., M. Ghayour-Mobarhan, H.A. Esmaeili, S.M.R. Parizadeh and S.J. Hosseini et al., 2009. Newly detected hypertension in an Iranian population: An epidemiological study. Asian Biomed., 3: 653-662.

Bahonar, B., N. Sarrafzadegan, K. Kelishadi, S. Shirani and M.A. Ramezani et al., 2011. Association of socioeconomic profiles with cardiovascular risk factors in Iran: The Isfahan healthy heart program. Int. J. Public Health, 56: 37-44. DOI: $10.1007 / \mathrm{s} 00038-010-0125-8$

Bonakdaran, S., S. Ebrahimzadeh and S.H. Noghabi, 2011. Cardiovascular disease and risk factors in patients with type 2 diabetes mellitus in Mashhad, Islamic Republic of Iran. East Mediterr Health J., 17: 640-646. PMID: 22259913

Boutayeb, A. and S. Boutayeb, 2005. The burden of non communicable diseases in developing countries. Int. J. Equity Health, 4: 2-2. DOI: 10.1186/1475-9276-4-2 
Delavari, A., M.H. Forouzanfar, S. Alikhani, A. Sharifian and R. Kelishadi, 2009. First nationwide study of the prevalence of the metabolic syndrome and optimal cutoff points of waist circumference in the Middle East: The national survey of risk factors for noncommunicable diseases of Iran. Diabetes Care, 32: 1092-1097. DOI: $10.2337 / \mathrm{dc} 08-1800$

Ebrahsim, S. and G. Davey-Smith, 2001. Exporting failure? Coronary heart disease and stroke in developing countries. Int. J. Epidemiol., 30: 201-205. DOI: 10.1093/ije/30.2.201

Elasmi, M., M. Feki, H. Sanhaji, R. Jemaa and S. Haj Taeib et al., 2009. Prevalence of conventional cardiovascular risk factors in the Great Tunis population. Rev. Epidemiol. Sante Publique., 57: 87-92. DOI: $10.1016 /$ j.respe.2008.12.010

EPDETHBCA, 2001. Executive summary of the third report of the National Cholesterol Education Program (NCEP) expert panel on detection, evaluation and treatment of high blood cholesterol in adults (Adult treatment panel III). JAMA, 285: 2486-97. DOI: 10.1001/jama.285.19.2486

Gupta, R., M. Sarna, J. Thanvi, V. Sharma and V.P. Gupta, 2007. Fasting glucose and cardiovascular risk factors in an urban population. J. Assoc. Physicians India, 55: 705-709. PMID: 18173023

Hadaegh, F., G. Shafiee, M. Hatami and F. Azizi, 2012. Systolic and diastolic blood pressure, mean arterial pressure and pulse pressure for prediction of cardiovascular events and mortality in a Middle Eastern population. Blood Press, 21: 12-18. DOI: $10.3109 / 08037051.2011 .585808$

Hadi, N. and N. Rostami-Gooran, 2004. Determinant factors of medication compliance in hypertensive patients of Shiraz, Iran. Arch. Iranian Med., 7: 292-296.

Jahromi, A.S., M. Shojaei, F. Mehdizadeh, S. Erfanian and A. Madani, 2014. A comparative study of antiphosphatidyl inositole antibodies in patients with myocardial infarction and healthy subjects. Am. J. Immunol., 10: 10-13. DOI: 10.3844/ajisp.2014.10.13

Jahromi, A.S., M. Shojaei, M.R. Farjam and A. Madani, 2013a. Anti-phosphatidylserine antibodies in acute myocardial infarction. Am. J. Immunol., 9: 96-100. DOI: 10.3844/ajisp.2013.96.100

Jahromi, A.S., M. Shojaei, M.R. Farjam and A. Madani, 2013b. Association of anti-phosphatidylcholine antibodies with acute myocardial infarction: A comparative study. Am. J. Immunol., 9: 116-119. DOI: 10.3844/ajisp.2013.116.119

Jahromi, A.S., M. Shojaei, M.R. Farjam and A. Madani, 2013c. The presence of anti-phosphatidylethanolamine antibodies in acute myocardial infarction. OnLine J. Biological Sci., 13: 126-130.

DOI: 10.3844/ojbsci.2013.126.130
Jahromi, A.S., M. Shojaie and A. Madani, 2010a. Cardiotrophin-1 in patients with acute myocardial infarction. Am. J. Applied Sci., 7: 1190-1194. DOI: 10.3844/ajassp.2010.1190.1194

Jahromi, A.S., M. Shojaie, S. Dana and A. Madani, 2010b. Anti-cardiolipin antibody in acute myocardial infarction. Am. J. Immunol., 6: 11-14. DOI: 10.3844 /ajisp.2010.11.14

Latheef, S.A. and G. Subramanyam, 2007. Prevalence of coronary artery disease and coronary risk factors in an urban population of Tirupati. Indian Heart J., 59: 157-164. PMID: 19122250

Meaney, E., A. Lara-Esqueda, G.M. Ceballos-Reyes, J. Asbun and A. Vela et al., 2007. Cardiovascular risk factors in the urban Mexican population: The FRIMEX study. Public Health, 121: 378-384. DOI: $10.1016 /$ j.puhe.2006.11.008

Murphy, B.P., T. Stanton and F.G. Dunn, 2009. Hypertension and myocardial ischemia. Med. Clin. North Am., 93: 681-695. DOI: 10.1016/j.mcna.2009.02.003

Nabipour, I., N.M. Amiri, S.R. Imami, S.M. Jahfari and A. Nosrati et al., 2008. Unhealthy lifestyles and ischaemic electrocardiographic abnormalities: The Persian Gulf healthy heart study. Eastern Mediterranean Health J., 14: 858-868. PMID: 19166169

NIH, 2008. The seventh report of the joint national committee on prevention, detection, evaluation and treatment of high blood pressure (JNC 7). National Institutes of Health.

Njelekela, M.A., R. Mpembeni, A. Muhihi, N.L. Mligiliche and D. Spiegelman et al., 2009. Genderrelated differences in the prevalence of cardiovascular disease risk factors and their correlates in urban Tanzania. BMC Cardiovascular Disorders, 9: 30-37. DOI: 10.1186/1471-2261-9-30

Pourahmad, M., A.S. Jahromy and M. Shojaei, 2009. Association of mycoplasma pneumoniae infection with myocardial infarction. Am. J. Immunol., 5: 84-88. DOI: 10.3844/ajisp.2009.84.88

Rahmanian, K. and M. Shojaie, 2011. Prevalence of metabolic syndrome in an adult urban population in the south of Iran. Iranian J. Diabetes Obesity, 3: 77-82.

Rahmanian, K. and M. Shojaie, 2012. The prevalence of pre-hypertension and its association to established cardiovascular risk factors in south of Iran. BMC Res. Notes, 5: 386. DOI: 10.1186/1756-0500-5-386

Rahmanian, K., M. Shojaei and A.R. Sotoodeh-Jahromi, 2013. Relation of type 2 diabetes mellitus with gender, education and marital status in an Iranian urban population. Reports Biochem. Molecular Biol., 1: 1-5.

Sharifi, F. Sharifi, S.N. Mousavinasab, M. Saeini and M. Dinmohammadi, 2009. Prevalence of metabolic syndrome in an adult urban population of the west of Iran. Experimental Diabetes Res., 2009: 1-5. DOI: $10.1155 / 2009 / 136501$ 
Shojaei, M., A.S. Jahromi, H. Ebadat, S.H. Moosavy and B. Seddigh et al., 2011. Anti- 32 glycoprotein-I antibody in acute myocardial infarction. Am. J. Applied Sci., 8: 758-761. DOI: 10.3844/ajassp.2011.758.761

Shojaie, M., A. Sotoodah and G. Shafaie, 2009a. Is adiponectin associated with acute myocardial infarction in Iranian non obese patients? Lipids Health Dis., 8: 17-17. DOI: 10.1186/1476-511X-8-17

Shojaie, M., A. Sotoodah, S. Roozmeh, E. Kholoosi and S. Dana, 2009b. Annexin V and anti-Annexin V antibodies: Two interesting aspects in acute myocardial infarction. Thrombosis J., 7: 13-13. DOI: $10.1186 / 1477-9560-7-13$

Tang, W.H., A. Maroo and J.B. Young, 2004. Ischemic heart disease and congestive heart failure in diabetic patients. Med. Clin. North Am., 88: 1037-1061. DOI: $10.1016 /$ j.mcna.2004.04.008

Tesfaye, F., P. Byass and S. Wall, 2009. Population based prevalence of high blood pressure among adults in Addis Ababa: Uncovering a silent epidemic. BMC Cardiovascular Disorders, 9: 39-48. DOI: $10.1186 / 1471-2261-9-39$

Wang, K.L., H.M. Cheng, S.Y. Chuang, H.A. Spurgeon and C.T. Ting et al., 2009. Central or peripheral systolic or pulse pressure: Which best relates to target organs and future mortality? J. Hypertens., 27: 461-467.
WHO, 2000. Obesity: Preventing and managing the global epidemic. Report of a WHO consultation. World Health Organ, Tech. Rep. Ser.

Willey, J.Z., Q. Xu, B. Boden-Albala, M.C. Paik and Y.P. Moon et al., 2009. Lipid profile components and risk of ischemic stroke: The Northern Manhattan Study (NOMAS). Arch. Neurol., 66: 1400-1406. DOI: 10.1001/archneurol.2009.210

Wilson, P.W., W.P. Castelli and W.B. Kannel, 1987. Coronary risk prediction in adults (the Framingham Heart Study). Am. J. Cardiol., 91: 91-94. DOI: 10.1016/0002-9149(87)90165-2

Wu, D.M., L. Pai, N.F. Chu, P.K. Sung and M.S. Lee et al., 2011. Prevalence and clustering of cardiovascular risk factors among healthy adults in a Chinese population: The MJ health screening center study in Taiwan. Int. J. Obesity, 25: 1189-1195.

Yang, Z.J., J. Liu, J.P. Ge, L. Chen and Z.G. Zhao et al., 2012. Prevalence of cardiovascular disease risk factor in the Chinese population: The 2007-2008 China national diabetes and metabolic disorders study. Eur. Heart J., 33: 213-220.

DOI: 10.1093/eurheartj/ehr205 